\title{
The impact of being of the female gender for household head on the prevalence of food insecurity in Ethiopia: a systematic- review and meta-analysis
}

\author{
Ayenew Negesse ${ }^{1,2^{*}}$ D, Dube Jara ${ }^{3,4}$, Habtamu Temesgen ${ }^{2}$, Getenet Dessie ${ }^{5}$, Temesgen Getaneh ${ }^{6}$, \\ Henok Mulugeta ${ }^{7}$, Zeleke Abebaw ${ }^{8}$, Tesfahun Taddege ${ }^{9}$, Fasil Wagnew ${ }^{7}$ and Yilkal Negesse ${ }^{10}$
}

\author{
* Correspondence: \\ ayenewnegesse@gmail.com \\ 'School of Human Nutrition, Food \\ Science and Technology, Hawassa \\ University, Hawassa, Ethiopia \\ ${ }^{2}$ Department of Human Nutrition \\ and Food Sciences, College of \\ Health Science, Debre Markos \\ University, Debre Markos, Ethiopia \\ Full list of author information is \\ available at the end of the article
}

\begin{abstract}
Background: Ethiopia signed both for Millennium Development Goals (MDGs) previously and Sustainable Development Goals (SDGs) currently to improve food security through gender equality and empowerment of women by positioning them as household leader. However, there is no concrete evidence about the impact of being of the female gender for household head on the prevalence of food insecurity at the national level, the authors' intention being to fill this gap.

Methods: Preferred Reporting Items for Systematic Reviews and Meta-Analyses protocol (PRISMA-P) guideline was followed. All major databases such as PubMed/ MEDLINE, WHOLIS, Cochrane Library, Embase, PsycINFO, ScienceDirect, Web of science, and reference lists were used to identify published articles, whereas shelves, author contact, Google, and Google Scholar were also searched to identify unpublished studies. Joanna Briggs Institute Meta-Analysis of Statistical Assessment and Review Instrument (JBI-MAStARI) was used for critical appraisal of studies. Metaanalysis was conducted using the STATA software version 14 . The random effect model was used to estimate the pooled prevalence of food insecurity at 95\% confidence level, while subgroup analysis and meta-regression were employed to identify the possible source of heterogeneity and the associated factors respectively. Moreover, Begg's test was used to check publication bias.

Results: A total of 143 articles were identified, of which 15 studies were included in the final model with a total sample size of 2084 female-headed households. The pooled estimate of food insecurity among female-headed households was $66.11 \%$ (95\% confidence level (CL) 54.61, 77.60). Female-headed households had 1.94 (95\% $C L 1.26$, 3.01) times the odds of developing food insecurity as compared with maleheaded households in Ethiopia. However, considerable heterogeneity across studies was also exhibited $\left(P^{2}=92.5 \%, p\right.$ value $\left.<0.001\right)$.

(Continued on next page)
\end{abstract}

\section{$\triangle B M C$}

(c) The Author(s). 2020 Open Access This article is licensed under a Creative Commons Attribution 4.0 International License, which permits use, sharing, adaptation, distribution and reproduction in any medium or format, as long as you give appropriate credit to the original author(s) and the source, provide a link to the Creative Commons licence, and indicate if changes were made. The images or other third party material in this article are included in the article's Creative Commons licence, unless indicated otherwise in a credit line to the material. If material is not included in the article's Creative Commons licence and your intended use is not permitted by statutory regulation or exceeds the permitted use, you will need to obtain permission directly from the copyright holder. To view a copy of this licence, visit http://creativecommons.org/licenses/by/4.0/. The Creative Commons Public Domain Dedication waiver (http://creativecommons.org/publicdomain/zero/1.0/) applies to the data made available in this article, unless otherwise stated in a credit line to the data. 
(Continued from previous page)

Conclusion: This review found that severity of food insecurity among female-headed households in Ethiopia was a more pronounced issue as compared with the general national estimate of food insecurity. Food insecurity among them was two-fold increased as compared with their men counterparts.

So that, the government of Ethiopia needs to outlook how cultural and social restriction of women's involvement in every aspect of activity affects their level of household food security. Beyond this, previous success and current gap of food insecurity among female-headed households should be explored in future research to run in accordance with Sustainable Development Goals (SDGs) specially with goals 2 and 5.

Keywords: Ethiopia, Female-headed households, Food insecurity, Meta-analysis, Systematic review

\section{Introduction}

The concept of food insecurity is explained by the quantity and quality of the available food, uncertainty about accessibility of food, and experiences of going hungry [1]. This concern is also experienced at the individual level with the issue of food consumption, allocation, and the physiological sensation of hunger [2].

Food insecurity can be measured by using the Food and Agriculture Organization (FAO) method for estimating calories available per capita at the national level, household income, and expenditure surveys; individual's dietary intake; anthropometry; and experience-based food insecurity measurement scales [3]. However, the commonly used measurement tools of food insecurity in Ethiopia are Household Food Insecurity Access Scale (HFIAS) and Household Hunger Scale (HHS). The HFIAS was developed by the Food and Nutrition Technical Assistance Project, Academy for Educational Development 2007 tool [4], whereas the HHS was also developed by Food and Nutrition Technical Assistance III Project in collaboration with USAID and Tufts University [5].

According to the UN World Food Program report of 2017, 108 million people were faced with a severe form of food insecurity [6]. There were also 815 million people faced with hunger though there were enough food produced to feed the population globally [7].

The issue of household food insecurity is more pronounced in sub-Saharan African (SSA) countries, including Ethiopia. The dramatic change of global warming, slow development of the global economy, and regional conflicts have a tremendous role on food insecurity in SSA [8].

From SSA countries including Ethiopia, Food and Agriculture Organization reported that more than one in ten households were affected with food insecurity in the year 2016 [9], whereas in the year 2017, 5.6 million people needed emergency food support [10]. To combat the issue of food insecurity and other cross-cutting issues, Ethiopia adopted both the Millennium Development Goals (MDGs) in the past and Sustainable Development Goals (SDGs) currently [11, 12].

In spite of the challenges, the SDGs agendas of 2030 are now under implementation in Ethiopia. Lack of availability of data in terms of information revolution as part of women's empowerment and food security in the developing countries in particular is a major constraint for monitoring and evaluation towards the progress of the activities and the ongoing outputs $[13,14]$. The approaches of SDGs also usually lack an 
underlying theoretical foundation; so that, beyond the political commitment of each United Nation (UN) country on the SDGs, each strategic objective of SDGs should be re-designed through baseline theoretical or conceptual framework with inclusive hypothesis [15].

Once more, SDGs are long-term development agendas and have the potential to be exposed to unexpected negative outcomes [16].

Finally, being universal transformation agenda itself, people-centered and nature of comprehensiveness may also affect future successfulness of each strategic goal of SDGs [17]. Hence, special mechanisms tailored for equitable resource distribution along with alternative strategic plans and approaches across national and sub-regional level like in Ethiopia are warranted to meet the SDGs in 2030. Having all those challenges, Ethiopia as part of Africa is using gender equality and empowerment of women as a tool for combating hunger and achieving food security [18]. Women empowerment and gender equality is not only a tool and a human right, but also it is a necessary foundation for sustainable development for a given country too [19].

In fact, giving power to women to increase productivity and food security is also ascertained as a mechanism for gender equality and empowerment of women [20] though it is a more broad term in different contexts [21].

In Ethiopia, there are pocket and fragmented studies across regions that explore the relationship between being of the female gender for household head and food security [22-36]. However, the studies were inconclusive and there is no concrete scientific evidence established at national level. Therefore, the main objective of this study was to identify the impact of being of the female gender for household head on the prevalence of food insecurity in Ethiopia as part of SSA.

Hence, this review is helpful to identify the basic factors for such devastating issue and to undertake early measures for the further achievement of SDGs in particular of goals 2 and 5 .

\section{Methods}

\section{Search strategy}

Initially databases were searched for both systematic review and meta-analysis to avoid duplications. First, DARE database (http://www.library.UCSF.edu) was explored in an attempt to confirm whether systematic review or meta-analysis exists and for availability of ongoing projects related to the topic. We also searched the two trial registries: ICTRP and Clinical Trials.Gov (searched December 2017). By using this method, it was confirmed that there was no review nor meta-analysis conducted linked to this topic.

All major databases such as PubMed/MEDLINE, WHOLIS, Cochrane Library, Embase, PsycINFO, ScienceDirect, Web of science, and reference lists were used to identify published articles, whereas shelves, author contact, Google, and Google Scholar were also used to identify unpublished studies. Our search for the published articles was restricted by the household head (female-headed households) and by country (studies conducted in Ethiopia only). All published and unpublished articles up to January 16, 2018, were included under this systematic review and meta-analysis. We followed the Preferred Reporting Items for Systematic Reviews and Meta-Analyses protocol (PRISMA-P) guideline for this meta-analysis [37]. 
The key terms used in building the search strategy were prevalence, magnitude, food security, food insecurity, female-headed household, and Ethiopia. The key terms were combined using Boolean operators to search the electronic databases. In addition, all fields and mesh terms were used while we used the advanced PubMed searching strategy.

\section{Eligibility criteria}

\section{Inclusion Criteria}

This systematic review and meta-analysis considered all the studies which were conducted both in urban and rural parts of Ethiopia that reported numbers of male-headed households, female-headed households, and who experienced food insecurity. To estimate the pooled prevalence, only female-headed individuals were included, whereas to identify the association between being of the female gender for household head and food insecurity, articles which reported food insecurity both at female- and male-headed households were considered. This systematic review and meta-analysis were not restricted based on publication conditions, publication time, and study designs.

\section{Exclusion Criteria}

Based on the eligibility criteria, we read their titles and abstracts. If studies were relevant for our review, we examined the full texts. Those papers not fully accessed at the time of our search process were excluded after contact was attempted with the principal investigator through email at least two times. The reason for the exclusion of these articles is that we were unable to assess the quality of each article in the absence of their full texts. Moreover, studies which did not report our outcome of interest were excluded after reviewing their full texts. Studies which did not report food insecurity both at female- and male-headed households at the same time were also excluded from the final meta-analysis. Once more, studies with poor quality according to the settled criteria were also excluded from this systematic review and meta-analysis.

Quality assessment

The database search results were combined and duplicate articles were removed manually using Endnote (version X8). Joanna Briggs Institute Meta-Analysis of Statistics Assessment and Review Instrument (JBI-MAStARI) adapted for cross-sectional study design was used [38]. Four independent reviewers critically appraised each individual paper. Disagreements between those reviewers were solved by discussion. If not, a fifth reviewer was involved to resolve inconsistencies among them. Studies which scored 50\% and above were included under this systematic review and meta-analysis.

\section{Data extraction}

Data were extracted by the four authors using a standardized data extraction spread sheet. The data extraction spreadsheet was piloted on 5 randomly selected papers and modified accordingly. The data extraction sheet included study characteristics such as the following: (1) authors' name, year, region, study or publication year, study design, study setting, and study population; (2) proportion of food insecurity; (3) information on the number of households who experienced either household food insecurity or 
security both from male- and female-headed households; and (4) studies' quality score and sampling techniques.

\section{Outcome measurement}

This study had one main outcome. It was household food insecurity among femaleheaded households. This was calculated as the number of female-headed households who were in food insecurity divided by total female-headed households and then multiplied by $100 \%$.

\section{Data analysis and synthesis}

The extracted data were entered into the computer using excel sheet and imported to STATA 14 for analysis. Evidence of publication bias and heterogeneity was assessed. Begg's test with $p$ value of less than 0.05 as a cutoff point to declare the presence of publication bias was considered [39]. Heterogeneity across studies was checked using Cochran Q statistic with the inverse variance $\left(I^{2}\right)$ value ranges from 30 to $60 \%, 50$ to $90 \%$, and 75 to $100 \%$ with moderate, substantial, and considerable heterogeneity across individual studies [40]. The forest plot was also used to visualize the presence of heterogeneity. A $p$ value less than 0.05 was also used to declare the presence of heterogeneity across studies. Potential differences between the studies were explored by subgroup analysis and metaregression. The findings were presented using forest plot with respective odds ratio and 95\% confidence intervals via random effects meta-analysis (DerSimonian and Laired) model to estimate the summative effect about the impact of being of the female gender for household head on the prevalence of food insecurity. The impact of heterogeneity across studies on the meta-analysis was quantified by $I^{2}$ statistic's tau and a cutoff point of $50 \%$ was used to declare substantial heterogeneity. The effect size of categorical data was expressed using odds ratio.

\section{Results}

\section{Search results}

A total of 143 studies were retrieved through our searches. Of these articles, 67 duplicated articles were excluded. From the remaining 76 articles, 39 articles were excluded after reading of titles and abstracts. Finally, 37 full-text articles were accessed and assessed for eligibility criteria. Based on the pre-defined criteria and after critical appraisal, only 15 articles were included in the final analysis (Fig. 1).

\section{Characteristics of the original articles}

A total of 15 original studies were included under this systematic review and metaanalysis. All the included studies were cross sectional and had a pooled sample size of 2084 households (see Table 1). The minimum sample size (3 households) was used in a study conducted at Oromia regional state [27], whereas maximum sample size (581 households) corresponded to Tigray regional state [30].

Of the included studies, four studies were conducted in Amhara region [22, 24-26], four studies in Oromia region [23, 27-29], two studies conducted in Tigray region [30, 31], and five studies in SNNPR region [32-36]. Regarding their publication conditions, five studies $[22,25,30,31,36]$ were gray literatures, whereas the remaining 11 studies 


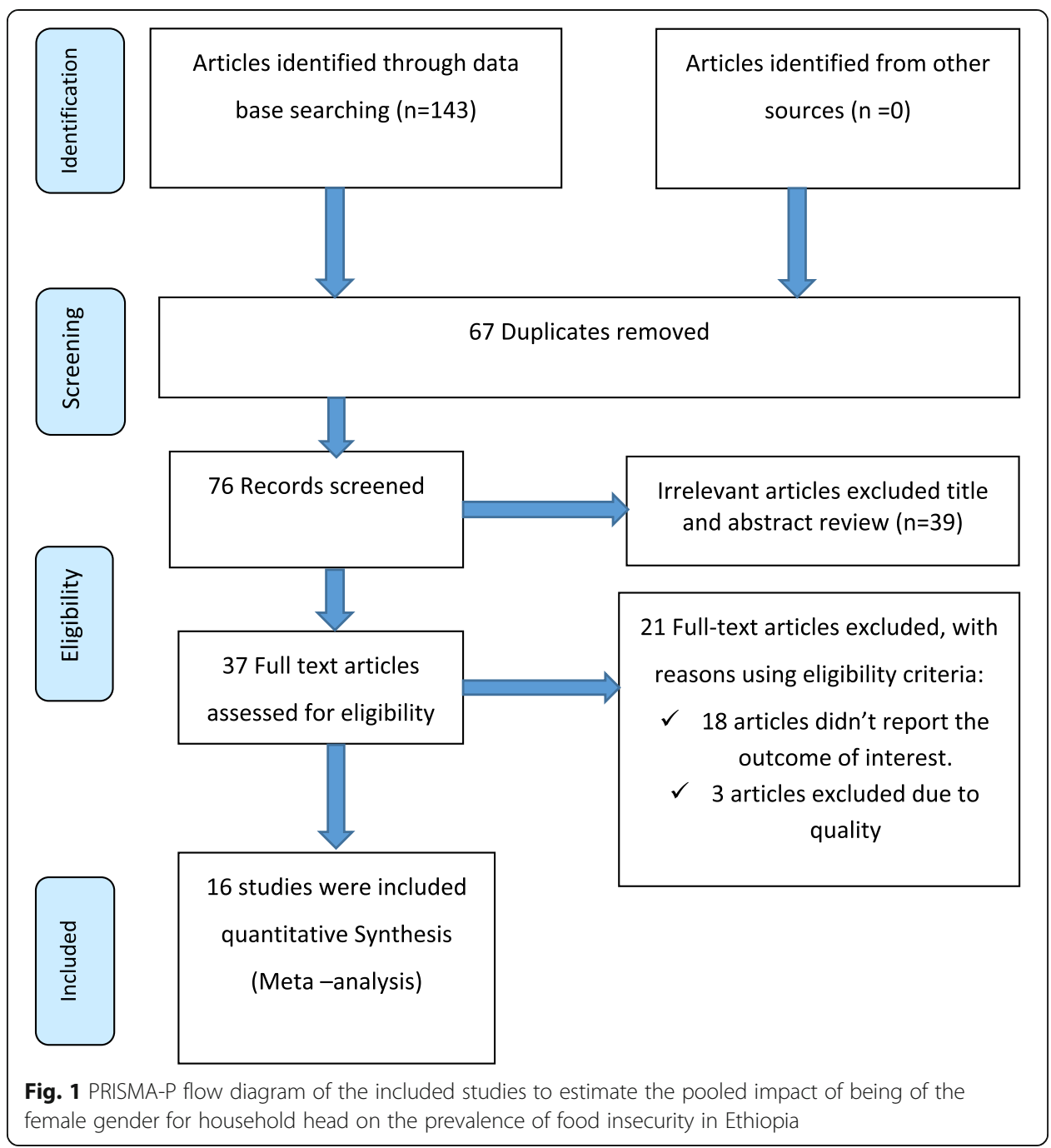

[23, 24, 26-29, 32-35] were published in reputable journals. After peer and independent evaluations of individual studies, all studies scored in the range from 5 up to 8 out of 9 values (Table 1$)$.

\section{Meta-analysis}

As indicated in the forest plot (Fig. 2), the pooled prevalence of food insecurity among female-headed households in Ethiopia was 66.11\% (95\% CI 54.61, 77.60). However, considerable heterogeneity was observed across studies $\left(I^{2}=96.8 \%, p\right.$ value $\left.=0.001\right)$. By considering this fact, random effect analysis was conducted.

\section{Sensitivity analysis}

Sensitivity analysis was also done to identify outlier studies, but there was no any individual study which influenced the overall pooled estimate of this systematic review and meta-analysis. 
Table 1 Characteristics of included studies to estimate the pooled prevalence among femaleheaded households in Ethiopia from 2007 up to 2017

\begin{tabular}{|c|c|c|c|c|c|c|c|c|}
\hline Region & Author & Year & $\begin{array}{l}\text { Study } \\
\text { setting }\end{array}$ & $\begin{array}{l}\text { Study } \\
\text { design }\end{array}$ & $\begin{array}{l}\text { Sample } \\
\text { size }\end{array}$ & $\begin{array}{l}\text { Type of } \\
\text { literature }\end{array}$ & $\begin{array}{l}\text { Quality } \\
\text { score }\end{array}$ & $\begin{array}{l}\text { Prevalence } \\
(95 \% \mathrm{Cl})\end{array}$ \\
\hline Amhara & Motbainor et al. [24] & 2016 & community & $\begin{array}{l}\text { Cross } \\
\text { sectional }\end{array}$ & 291 & Article & 8 & $\begin{array}{l}55.67(49.96, \\
61.38)\end{array}$ \\
\hline Amhara & Alem [22] & 2016 & Community & $\begin{array}{l}\text { Cross } \\
\text { sectional }\end{array}$ & 49 & Gray & 6 & $\begin{array}{l}77.55(65.87, \\
89.23)\end{array}$ \\
\hline Amhara & Epherm [25] & 2008 & Community & $\begin{array}{l}\text { Cross } \\
\text { sectional }\end{array}$ & 17 & Gray & 5 & $\begin{array}{l}76.47(56.31, \\
96.63)\end{array}$ \\
\hline Amhara & Motbainor et al. [24] & 2016 & community & $\begin{array}{l}\text { Cross } \\
\text { sectional }\end{array}$ & 277 & Article & 8 & $\begin{array}{l}66.43(60.86, \\
71.99)\end{array}$ \\
\hline Amhara & Endale et al. [26] & 2014 & Community & $\begin{array}{l}\text { Cross } \\
\text { sectional }\end{array}$ & 115 & Article & 8 & $\begin{array}{l}92.17(87.27, \\
97.08)\end{array}$ \\
\hline Oromia & $\begin{array}{l}\text { Mequanent and } \\
\text { Esubalew [28] }\end{array}$ & 2015 & Community & $\begin{array}{l}\text { Cross } \\
\text { sectional }\end{array}$ & 23 & Article & 5 & $\begin{array}{l}69.57(50.76, \\
88.37)\end{array}$ \\
\hline Oromia & $\begin{array}{l}\text { Beyene and Muche } \\
\text { [23] }\end{array}$ & 2010 & Community & $\begin{array}{l}\text { Cross } \\
\text { sectional }\end{array}$ & 24 & Article & 5 & $\begin{array}{l}66.67(47.83, \\
85.53)\end{array}$ \\
\hline Oromia & Mitiku et al. [29] & 2012 & Community & $\begin{array}{l}\text { Cross } \\
\text { sectional }\end{array}$ & 27 & Article & 5 & $\begin{array}{l}40.74(22.21, \\
59.27)\end{array}$ \\
\hline Oromia & $\begin{array}{l}\text { Mequanent et al. } \\
\text { [27] }\end{array}$ & 2014 & Community & $\begin{array}{l}\text { Cross } \\
\text { sectional }\end{array}$ & 3 & Article & 5 & $\begin{array}{l}66.67(46.50, \\
86.83)\end{array}$ \\
\hline Tigray & Eyob [30] & 2012 & Community & $\begin{array}{l}\text { Cross } \\
\text { sectional }\end{array}$ & 581 & Gray & 8 & $\begin{array}{l}32.19(28.39, \\
35.98)\end{array}$ \\
\hline Tigray & Tsegay [31] & 2009 & Community & $\begin{array}{l}\text { Cross } \\
\text { sectional }\end{array}$ & 251 & Gray & 8 & $\begin{array}{l}49.00(42.82, \\
55.19)\end{array}$ \\
\hline SNNP & Shone et al. [32] & 2017 & Community & $\begin{array}{l}\text { Cross } \\
\text { sectional }\end{array}$ & 143 & Article & 8 & $\begin{array}{l}81.82(75.50, \\
88.14)\end{array}$ \\
\hline SNNP & Leza and Kuma [34] & 2015 & Community & $\begin{array}{l}\text { Cross } \\
\text { sectional }\end{array}$ & 100 & Article & 6 & $\begin{array}{l}76.00(67.63, \\
84.37)\end{array}$ \\
\hline SNNP & Tantu et al. [33] & 2017 & Community & $\begin{array}{l}\text { Cross } \\
\text { sectional }\end{array}$ & 121 & Article & 7 & $\begin{array}{l}65.29(56.81, \\
73.77)\end{array}$ \\
\hline SNNP & $\begin{array}{l}\text { Fikire and Bekele } \\
\text { [36] }\end{array}$ & 2014 & Community & $\begin{array}{l}\text { Cross } \\
\text { sectional }\end{array}$ & 41 & Gray & 5 & $\begin{array}{l}75.61(62.46, \\
88.75)\end{array}$ \\
\hline SNNP & $\begin{array}{l}\text { Negussie and } \\
\text { Alemayehu [35] }\end{array}$ & 2013 & Community & $\begin{array}{l}\text { Cross } \\
\text { sectional }\end{array}$ & 21 & Article & 5 & $\begin{array}{l}66.67(46.50, \\
86.83)\end{array}$ \\
\hline
\end{tabular}

\section{Subgroup analysis}

Since this review is accounted for considerable heterogeneity, subgroup analysis based on region, publication condition, type of tool used to measure household food insecurity, and sampling technique were considered to identify the possible source of heterogeneity across studies (Table 2). However, the subgroup analysis result indicated that the source of heterogeneity was not due to regional, publication condition, and sampling technique differences. As reported in the subgroup analysis, the lowest and highest prevalence of food insecurity were $40.41 \%$ (95\% CI 23.93, 56.89) and $74.18 \%$ (95\% CI 67.13, 81.23) at Tigray and South Nations, Nationalities and Peoples of Ethiopia (SNNPs) respectively (Table 2).

Beyond the subgroup analysis, publication bias was checked using Begg's test, though the value was find to be insignificant at $p$ value of 0.753 , which indicates there was no any study effect. 


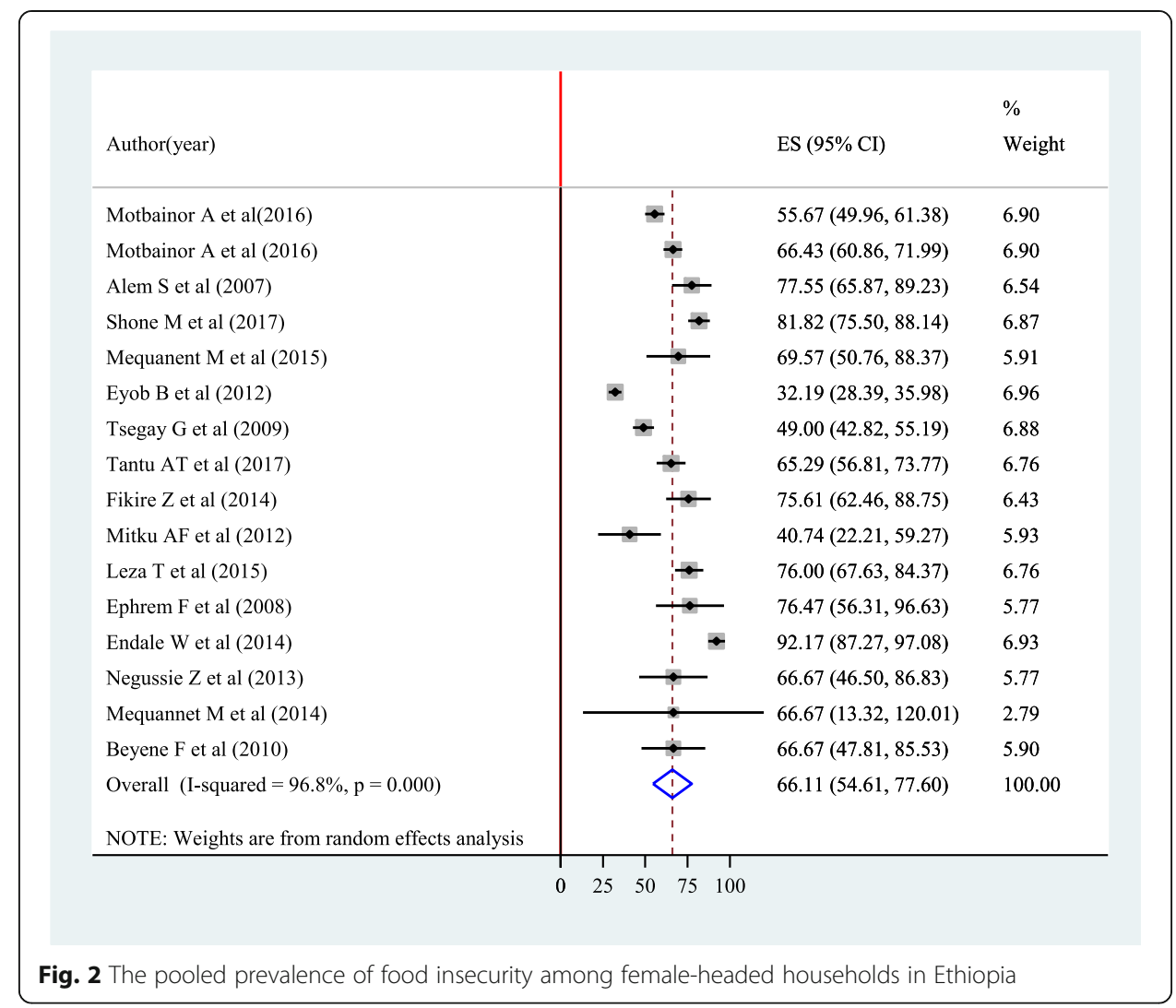

\section{Meta-regression}

Beyond the subgroup analysis, meta-regression was also undertaken by considering both continuous and categorical data. Region where the study was conducted, sample size, year, publication condition, sampling technique, and studies conducted either of the two development goals and measurement tool for food insecurity were considered in the meta-regression. However, all the candidate variables in the meta-regression showed that heterogeneity in the prevalence of food insecurity was not associated with sample size variation, the tool used by authors to measure food insecurity, regional

Table 2 Subgroup analysis of the pooled prevalence of food insecurity among female-headed households in Ethiopia from 2007 up to 2017

\begin{tabular}{|c|c|c|c|c|c|c|}
\hline Subgroup & & $\begin{array}{l}\text { Number of included } \\
\text { studies }\end{array}$ & $\begin{array}{l}\text { Prevalence } \\
(95 \% \mathrm{Cl})\end{array}$ & $\begin{array}{l}\text { Heterogeneity } \\
\text { statistics }\end{array}$ & $p$ value & $P^{2}$ \\
\hline \multirow[t]{4}{*}{ By region } & Amhara & 5 & $73.48(57.42,89.54)$ & 99.8 & $<0.001$ & 96.0 \\
\hline & Oromia & 4 & $59.48(43.93,75.02)$ & 5.65 & 0.130 & 46.9 \\
\hline & Tigray & 2 & $40.41(23.93,56.89)$ & 20.63 & $<0.001$ & 95.2 \\
\hline & SNNP & 5 & $74.18(67.13,81.23)$ & 10.16 & 0.038 & 60.6 \\
\hline \multirow{2}{*}{$\begin{array}{l}\text { By publication } \\
\text { condition }\end{array}$} & Published & 11 & $68.93(59.30,78.56)$ & 123.06 & $<0.001$ & 91.9 \\
\hline & Unpublished & 5 & $61.12(42.34,79.90)$ & 102.42 & $<0.001$ & 96.1 \\
\hline \multirow{2}{*}{$\begin{array}{l}\text { By sampling } \\
\text { method }\end{array}$} & Multi-stage & 13 & $70.87(62.46,79.29)$ & 123.15 & $<0.001$ & 90.3 \\
\hline & Survey & 3 & $47.07(31.05,63.10)$ & 29.99 & $<0.001$ & 93.3 \\
\hline \multirow{2}{*}{$\begin{array}{l}\text { By measurement } \\
\text { tool }\end{array}$} & HFIAS & 5 & $72.35(58.34,86.36)$ & 109.31 & $<0.001$ & 96.3 \\
\hline & $\mathrm{HHS}$ & 11 & $62.82(49.19,76.46)$ & 171.83 & $<0.001$ & 94.2 \\
\hline
\end{tabular}


differences, type of sampling technique, publication year, and publication condition (Table 3).

Three studies [22, 31, 32] indicated that there was significant association between being of the female gender for household head and food insecurity in Ethiopia, whereas the remaining studies [23-30,33-36] indicated that there was insignificant association between food insecurity and being of the female gender for household head. This indicated that the findings were not concrete and evidence for doing further systematic review and meta-analysis.

From this meta-analysis (Fig. 3), female-headed households had 1.94 (95\% CL 1.26, 3.01) times the odds of developing food insecurity as compared with male-headed households. However, considerable heterogeneity across studies was also exhibited $\left(I^{2}=\right.$ $92.5 \%, p$ value $<0.001)$.

\section{Discussion}

This review focused on the prevalence of food insecurity among female-headed households and the effect of gender for household on food insecurity in Ethiopia from 2007 [22] up to 2017 [32, 33]. This review included studies which were done after the initiation of MDGs up to 2 years after Ethiopia signed the SDGs.

We found that two out of the three female-headed households in Ethiopia faced food insecurity. This issue was almost double as compared with male-headed rural households. This is incompatible with SDG 2 in which gender parity was settled as means of ending hunger, achieving food security and improved nutrition, and promoting sustainable agriculture for the year 2030 [41]. If gender preference on household head is not eliminated, the prevalence of food insecurity would increase by $12-17 \%$ [42]. It is also $39.11 \%$ greater than the regional overview of food insecurity across Northeast and North African countries conducted in the year 2014-2016 among the general population [43], and two times greater the South Sudan's conducted on general population [44]. This discrepancy is more likely explained by geographic, social, cultural, and economic differences. Moreover, the current review considered only female-headed rural

Table 3 Meta-regression for the included studies to identify source of heterogeneity for the prevalence of food insecurity among female-headed rural households in Ethiopia from 2007 up to 2017

\begin{tabular}{llll}
\hline Variables & Characteristics & Coefficients & $p$ value \\
\hline Year & Publication year & -0106513 & 0.501 \\
Sample & Sample size & 1.54988 & 0.782 \\
Article & Un-published article & -14.3361 & 0.640 \\
Region & Amhara region & Reference & Reference \\
& Oromia & -17.28004 & 0.694 \\
& Tigray & -34.76256 & 0.425 \\
Sampling technique & Census survey & -22.95167 & 0.470 \\
& Multi-stage & Reference & Reference \\
Measurement tool & HFIAS & 15.07132 & 0.674 \\
& HFS & Reference & Reference \\
Development goals & SDGs & 5.126678 & 0.574 \\
& MDGs & Reference & Reference \\
\hline
\end{tabular}




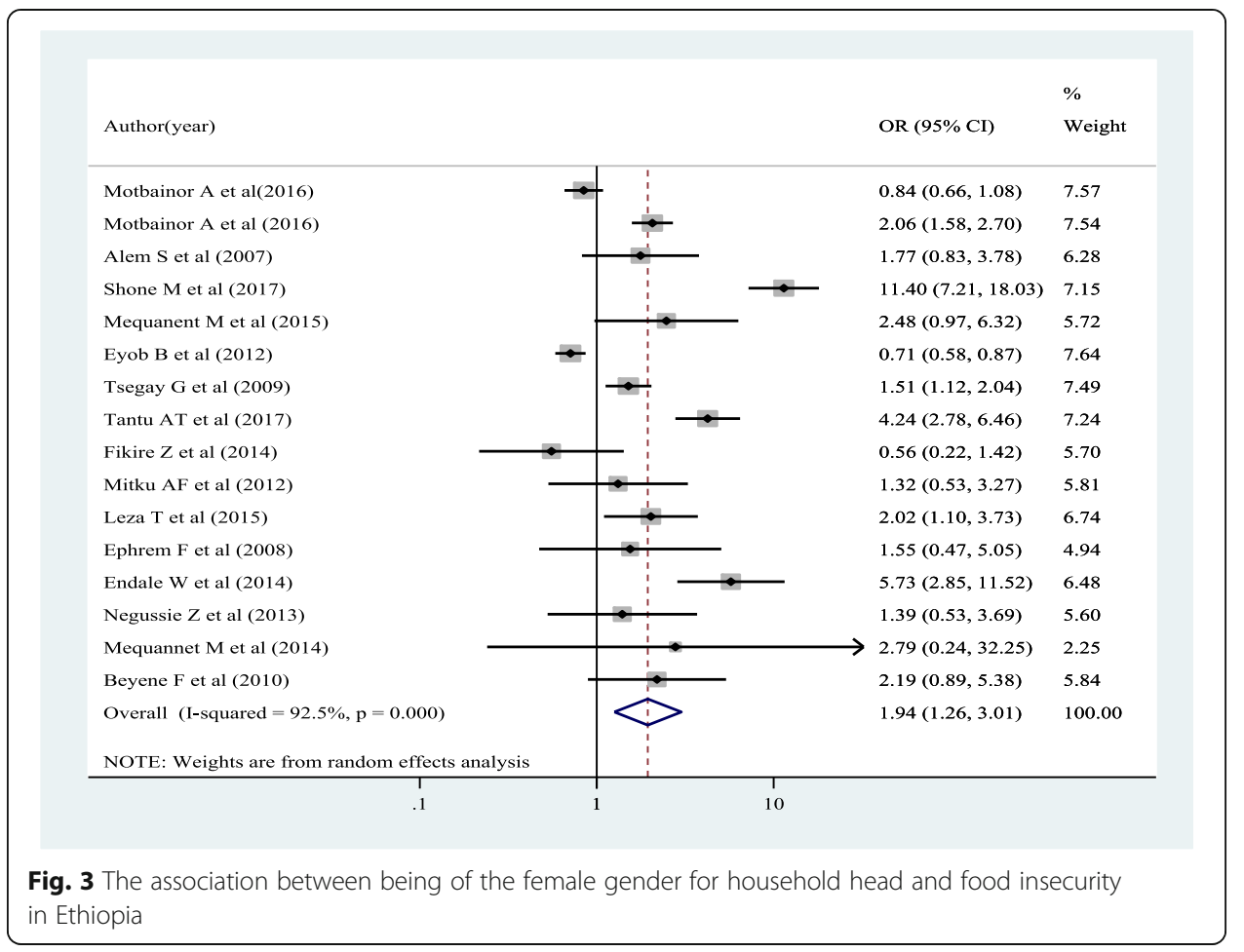

households. It is also six-fold greater than national estimates of food insecurity in Ethiopia [9]. This discrepancy may be due to study population differences and underestimated decision-making power of women [45].

Based on the subgroup analysis, Tigray was the most food secured region, whereas SNNPs were the least food secured region in Ethiopia. This can be explained by the well-connected community roads in Tigray region [46]. The relationship between food insecurity and public investment in road infrastructure was well established elsewhere [47]. Moreover, the human development index of Tigray region was relatively high as compared with other regions [48]. Human development index and food insecurity are interlinked to each other [49].

In comparison with male-headed households, female-headed households were more likely prone to food insecurity. Despite Ethiopia was one of the signatory countries to improve food security by implementing SDGs in particular of goals 2 and 5, the prevalence of food insecurity among female-headed households in Ethiopia was increased in the SDGs period as compared with the time of MDGs as showed in the subgroup analysis. So the previous success and the current flaws in food security among femaleheaded households should be explored in future research help to achieve all the SDGs in Ethiopia. This is supported by the systematic review and meta-analysis conducted both at global level and in other SSA countries [50-52]. This could be possibly explained by the fact that the decision-making power of women in the household may be undermined and women do not have the right to inherit their families capital [45] that coupled with their low income due to lower position in the labor force [52]. Beyond this, female-headed households also faced multiple challenges such as limited access to land ownership, market, and lack of agriculture extension services [53], and also because female-headed households cannot adopt new agricultural technologies, more 
prone to climatic changes $[54,55]$. This also again can be explained by the fact that female-headed households specially from the rural areas may lack natural resources and information which are very important to mitigate food insecurity [56, 57]. However, no significant differences in food insecurity were found in a study conducted in Kenya, Uganda, and Tanzania [58] despite reasons were not provided. No significant differences of food insecurity among women and men were also found in a study conducted at Bangladesh. This can be explained because between both genders may have freedom to participate in the labor force without any social and cultural restrictions [59].

Our study has several strengths and limitations. Primarily, this systematic review and meta-analysis used internationally accepted tools for critical appraisal system for quality assessment of individual studies. Secondly, it included both published and unpublished studies. Since there were no original studies reported from some regional states of Ethiopia, this systematic review and meta-analysis may not represent all female-headed households in Ethiopia. This review included only cross-sectional studies, in which longitudinal studies are strong enough to indicate the true cause-effect relationship between being of the female gender as household head and food security [60].

Time differences between individual studies, variation of tools to measure outcome variable, sample size, nature of data collected, and geographic location may affect the outcome. However, appropriate statistical model (random effect model) along with best statistical estimation approaches such as subgroup analysis and meta-regression were considered to narrow this limitation.

Once more, this systematic review and meta-analysis also appreciated that the nature of female individuals in their leadership style to cope with food insecurity may also affect the overall pooled estimate of food insecurity. Hence, this is also the interest of the authors to warrant further exploration about the relationship between leadership style among female-headed households and food security outcomes.

\section{Conclusion}

This systematic review and meta-analysis found that the prevalence of food insecurity among female-headed households was higher than the national estimate of household food insecurity and a significant association was found with gender as household head. Therefore, the government of Ethiopia should work to address cultural and social restrictions of women's involvement as if affects their level of household food security. As more than half of the Ethiopian population is female, previous success and current flaws in food security among female-headed households should be explored in future research immediately to run in accordance with the SDGs specially with goals 2 and 5 . Beyond this, it is also better to focus on the expansion of community roads and on activities which may increase human development index of the country in order to be contemplated with food security.

\footnotetext{
Abbreviations

FAO: Food and Agriculture Organization; HFIAS: Household Food Insecurity Access Scale; HHS: Household Hunger Scale; MDGs: Millennium Development Goals; PRISMA-P: Preferred Reporting Items for Systematic Reviews and MetaAnalyses protocols; SDGs: Sustainable Development Goals; SNNPs: South Nations Nationalities and Peoples; SSA: SubSaharan Africa
} 


\section{Authors' contributions}

$A N, Z A, T T, Y N, F W$, and TG developed the protocol and involved in the design, selection of study, data extraction, statistical analysis, and developing the initial drafts of the manuscript. GD, HT, and FW involved in quality assessment. $A N, Z A, H M$, and DJ prepared and revising subsequent drafts. AN, ZA, FW, YN, and HT prepared the subsequent and final draft of the manuscript. All authors read and approved the final draft of the manuscript.

\section{Funding}

No funding was obtained for this study.

\section{Availability of data and materials}

The data sets analyzed during the current study are available from the corresponding author upon reasonable request.

Ethics approval and consent to participate

Not applicable.

\section{Consent for publication}

Not applicable.

\section{Competing interests}

The authors have declared that there is no competing interest.

\section{Author details}

${ }^{1}$ School of Human Nutrition, Food Science and Technology, Hawassa University, Hawassa, Ethiopia. ${ }^{2}$ Department of Human Nutrition and Food Sciences, College of Health Science, Debre Markos University, Debre Markos, Ethiopia. ${ }^{3}$ Department of Public Health, College of Health Science, Debre Markos University, Debre Markos, Ethiopia. ${ }^{4}$ School of Public Health, College of Medicine and Health Science, Addis Ababa University, Addis Ababa, Ethiopia. ${ }^{5}$ Department of Nursing, College of Health Science, Bahir Dar University, Bahir Dar, Ethiopia. ${ }^{6}$ Department of Midwifery, College of Health Science, Debre Markos University, Debre Markos, Ethiopia. ${ }^{7}$ Department of Nursing, College of Health Science, Debre Markos University, Debre Markos, Ethiopia. ${ }^{8}$ Department of Health Informatics, University of Gondar, Gondar, Ethiopia. ${ }^{9}$ Ethiopia Field Epidemiology and Laboratory Training Program (EFELTP), University of Gondar, Gondar, Ethiopia. ${ }^{10}$ College of Medicine and Health Sciences, Mizan Tepi University, Mizan, Ethiopia.

Received: 17 September 2019 Accepted: 20 May 2020

Published online: 05 June 2020

\section{References}

1. Katherine A. Briefel Ronette R, and Frongillo Jr Edward A et al: Food insufficiency exists in the United States: results from the third National Health and Nutrition Examination Survey (NHANES III). American Journal of Public Health. 1998;88(3): 419-26.

2. Campbell Cathy C. and Desjardins Ellen: A model and research approach for studying the management of limited food resources by low income families. Journal of Nutrition Education. 1989;21(4):162-71.

3. Food and Agricalture Organization: Measurement and assessment of food deprivation and undernutrition [cited 2007 Feb 22.

4. Coates Jennifer, Swindale Anne, and Bilinsky Paula: Household Food Insecurity Access Scale (HFIAS) for measurement of food access: indicator guide: version 3. 2007.

5. FANTA III, USAID, and Tufts university: A guide for Household hunger scale (HHS) August, 2011

6. World Food Program: World Food program published on March 2017; available from https://www.wfp.org/news/newsrelease/108-million-people-world-face-severe-food-insecuritysituation-worsening.

7. Food and Agricalture Organization: The state of food and nutrition security in the world; available from http://www.fao. org/state-of-food-security-nutrition/en/. 2017

8. Food and Agricalture Organization: Africa Regional Overview of Food Security and Nutrition (The Food Security and Nutrition-Conflict Nexus: Building Resilience for Food Security, Nutrition and Peace), published Nov 2017.

9. Food and Agricalture Organization: Prevalence of food insecurity situation report in Ethiopia, february 2016

10. Food and Agriculture Organization (FAO): Household food insecurity situation in Ethiopia; a report from January 2017.

11. United Nations of Ethiopia: Adpotion of Millinum Development Goals (MDGs) which is available from http://et.one.un. org/content/unct/ethiopia/en/home/assistance-framework/millennium-development-goals.html.

12. United Nations in Ethiopia: Ethiopia adopted the Sustainable development Goals which is avilable at: https://ethiopia.un. org/en/sdgs.

13. Suriyanarayanan S. Addressing challenges of developing countries in implementing five priorities for sustainable development goals. Ecosystem Health and Sustainability. 2015;1(7):1-4.

14. Charlton Karen E: Food security, food systems and food sovereignty in the 21 st century: a new paradigm required to meet Sustainable Development Goals. 2016.

15. Bebbington J, Unerman J. Achieving the United Nations SDGs. Auditing \& Accountability Journal: An enabling role for accounting research Accounting; 2015.

16. Joy H, Larry M. Monitoring progress towards the Sustainable Development Goals. The Sustainable Development. 2015.

17. Greg S, Abbas R. Sustainable development and geospatial information: a strategic framework for integrating a global policy agenda into national geospatial capabilities. Geo-spatial information science. 2017;20(2):59-76.

18. Joel N, Roseline R, Susan K, et al. Integrating a broader notion of food security and gender empowerment into the African Green Revolution. Food Security. 2009;1(3):351-60.

19. United Nations. Gender equality and women's empowerment. United Nations Sustainable Development.

20. Sabina A, Ruth M-D, Amber P, et al. The women's empowerment in agriculture index. World Development. 2013;52:71-91. 
21. Solava I, Sabina A. Agency and empowerment: a proposal for internationally comparable indicators. Oxford development studies. 2007;35(4):379-403.

22. Shumiye A. Determinants of food insecurity in rural households in Tehuludere Woreda. South Wello Zone of the Amhara Region. Master's Thesis: Addis Ababa University, Ethiopia; 2007.

23. Fekadu B, Mequanent M. Determinants of food security among rural households of Central Ethiopia: an empirical analysis. Quarterly Journal of International Agriculture. 2010;49(4):299.

24. Achenef M, Alemayehu W, Abera K. Level and determinants of food insecurity in East and West Gojjam zones of Amhara Region, Ethiopia: a community based comparative cross-sectional study. BMC Public Health. 2016;16:503.

25. Fufa E. The link between food security and land degradation: analysis of determinants in drought prone area of north east Ethiopia: a case of Sekota Woreda. A master thesis presented to the school of graduate studies of AUA. 2008.

26. Worku E, Zelalem M, Azeb A, et al. Food insecurity in Farta District, Northwest Ethiopia: a community based crosssectional study. BMC Res Notes. 2014;7:130.

27. Muche M. Birara Endalew, and KorichoTesfalem: Determinants of household food security among Southwest Ethiopia rural households. Food Science and Technology. 2014;2(7):93-100.

28. Mequanent Muche, and EsubalewTadele: Analysis of household level determinants of food security in Jimma Zone, Ethiopia. Analysis 2015, 6(9).

29. Mitiku AF: B. and Tadesse, B. 2012."Empirical analysis of the determinants of rural households' food security in Southern Ethiopia. The case of Shashemene District". Basic Research Journal of Agricultural Science and Review, 1(5).

30. Eyob Bekele Juhar: Food security and vulnerability analysis at household level. Mekelle University; 2012.

31. Tsegay G. Determinants of food security in rural households of the Tigray region. Addis Ababa University, Ethiopia: Unpublished MPhil thesis; 2009.

32. Malla S, Tsegaye D, Bereket $Y$, et al. Household food insecurity and associated factors in West Abaya district, Southern Ethiopia, 2015. Agriculture \& Food Security. 2017;6(1):2.

33. Tadesse TA, Demissie GT, Kuma SB, et al. Household food insecurity and associated factors among households in Wolaita Sodo town, 2015. Agriculture \& Food Security. 2017;6(1):19.

34. Tekle L, Berhanu K. Determinants of rural farm household food security in Boloso Sore District of Wolaita Zone in Ethiopia. Asian J Agric Ext Econ Sociol. 2015;5(2):57-68.

35. Zerihun N, Getachew A. Levels of household food insecurity in rural areas of Guraghe zone. Southern Ethiopia. Journal of Agricultural Research. 2013;2.

36. ZELALEM FIKIRE, Abate B. Determinants of food security in the rural households of Meskan WoredA. Gurage Zone: SNNPR, Ethiopia. St. Mary's University; 2014.

37. David M, Alessandro L, Jennifer T, et al. Preferred reporting items for systematic reviews and meta-analyses: the PRISMA statement. Physical therapy. 2009;89(9):873-80.

38. Institute Jonna Briggs: Meta-analysis of statistics: assessment and review instrument (JBI Mastari). Adelaide: Joanna Briggs Institute. 2006:20032.

39. Begg Colin B, and Mazumdar Madhuchhanda: Operating characteristics of a rank correlation test for publication bias. Biometrics 1994:1088-1101.

40. collaboration C: Cochrane handbook for systematic reviews of interventions (Cochrane Book Series). In.: Version; 2008.

41. United Nations: The sustainable development goal 2 as "End hunger, achieve food security and improved nutrition and promote sustainable agriculture" available from https:/www.un.org/sustainabledevelopment/hunger/.

42. World Food program: The link between food insecurity and gender equality in the global persepective, available at http://www1.wfp.org/gender-equality.

43. Africa Regional Overview of Food Security and Nutrition (Building Resilience for Food Security and Nutrition in Times of Conflict and Crisis: A Perspective from the Near East and North Africa Region). 2017.

44. The Reliefweb: prevalence of food insecurity at South Sudan, an ongoing report from 2015-2018.

45. Hassen and Shitaye T: Food insecurity among female-headed households in ankober, northern Ethiopia. 2007.

46. Ali M, Meaza H. Geographical analysis of road transportation of Ethiopia. Ethiopian Journal of Environmental Studies and Management. 2015;8(1):846-5.

47. Robin H, Leonard W. Food security and public investment in rural infrastructure: some political economy considerations. UNDP African Human Development Report. 2012:2012-7.

48. Global Data Lab: subnational human development index which is available at https://hdi.globaldatalab.org/areadata/ shdi/. 2017.

49. Pedro C, Ricardo F-N, Leo H-P, et al. Food security and human development in Africa: strategic considerations and directions for further research. African Development Review. 2011;23(2):237-46.

50. Jung Natalie Miranda, de Bairros Fernanda Souza, and Pattussi Marcos Pascoal et al: Gender differences in the prevalence of household food insecurity: a systematic review and meta-analysis. Public health nutrition 2017, 20(5):902916.

51. Female-headed households continue to bear the brunt of poverty and ongoing food shortages in Zimbabwe, Zambia and Malawi, according to recent surveys: JOHANNESBURG, 28 July 2004.

52. Selepe Bolyn Mosa, Mtyingizane Samela Shalom, and FM Masuku: Factors contributing to household food insecurity in Mhlontlo area, Eastern Cape, South Africa. J Hosp Manage Tourism 2015, 4(1):2223-2814X.

53. Quisumbing Agnes R. and Pandolfelli Lauren: Promising approaches to address the needs of poor female farmers: resources, constraints, and interventions. World development. 2010;38(4):581-92.

54. Menale K. Ndiritu Simon Wagura, and Stage Jesper: What determines gender inequality in household food security in Kenya? Application of exogenous switching treatment regression. World Development. 2014;56:153-71.

55. Patti K, Henry N, Anja G, et al. Are food insecure smallholder households making changes in their farming practices? Evidence from East Africa. Food Security. 2012;4(3):381-97.

56. Elizabeth B, Claudia R, Barrack O, et al. Adapting agriculture to climate change in Kenya: household strategies and determinants. Journal of environmental management. 2013;114:26-35.

57. Branca Giacomo, Lipper Leslie, al aMNe: Food security, climate change, and sustainable land management. A review. Agronomy for sustainable development 2013, 33(4):635-650. 
58. Silvia S, Douxchamps S, Kristjanson P, et al. Households and food security: lessons from food secure households in East Africa. Agriculture \& Food Security. 2015;4(1):23.

59. Debdulal M, Mohammad R. Are female-headed households more food insecure? Evidence from Bangladesh. World development. 2010;38(4):593-605.

60. Joseph CE, Marius R, Jules H-S, et al. Longitudinal studies. Journal of thoracic disease. 2015;7(11):E537.

\section{Publisher's Note}

Springer Nature remains neutral with regard to jurisdictional claims in published maps and institutional affiliations.

Ready to submit your research? Choose BMC and benefit from:

- fast, convenient online submission

- thorough peer review by experienced researchers in your field

- rapid publication on acceptance

- support for research data, including large and complex data types

- gold Open Access which fosters wider collaboration and increased citations

- maximum visibility for your research: over $100 \mathrm{M}$ website views per year

At $\mathrm{BMC}$, research is always in progress.

Learn more biomedcentral.com/submissions 